\title{
Estimating Dependence Structure and Risk of Financial Market Crash
}

\author{
Ogunyiola Ayorinde Joshua ${ }^{1}$, Peter N. Mwita ${ }^{2} \&$ Carolyn N. Ngenja ${ }^{3}$ \\ ${ }^{1}$ Pan African University Institute for Basic Science, Technology and Innovation, Kenya \\ 2 Jomo Kenyatta University of Agriculture and Technology, Kenya \\ ${ }^{3}$ Srathmore University, Kenya \\ Correspondence: Ogunyiola Ayorinde Joshua, Pan African University Institute for Basic Sciences, Technology and \\ Innovation, Kenya. E-mail: ayoogunyiola@yahoo.com
}

\author{
Received: September 14, 2016 Accepted: September 26, 2016 Online Published: October 26, 2016 \\ doi:10.5539/ijsp.v5n6p85 \\ URL: http://dx.doi.org/10.5539/ijsp.v5n6p85
}

\begin{abstract}
In this paper, we estimate the dependence structure between international stock markets using copulas. Different relationships that exist in normal and extreme periods were estimated using Clayton copula. The Inference Functions for Margins method was used in estimating the clayton copula parameter thereby obtaining dependence estimates used in estimating Value-at-Risk. Extreme events are likely to alter the dependence structure of financial markets. This could have implications for investment decisions and ability to estimate the risk of financial markets crash. Results reveal that during the crisis period (2007-2009), maximum possible loss of market value is $75.9 \%$ and $77.6 \%$ with a confidence interval of 90\% for the Kenya-Nigeria and Kenya-South Africa portfolios respectively. This implies that the Kenya-South Africa portfolio has the highest risk.
\end{abstract}

Keywords: copula, asymmetric dependence, Value-at-Risk

\section{Introduction}

Dependence structure between random variables is crucial in multivariate analysis. In finance, dependence structure between financial markets are critical for investors, policymakers and researchers to make informed decision about investing their resources and making correct investment strategies (Ling, 2006). This is because, interest rates and equity prices move in opposite directions in normal periods but in periods of financial turmoil, they tend to co-move.

The extent of interconnectedness and interdependence of the financial system was highlighted during the 2007-2009 financial crisis (Aloui et al., 2011). The studies of Dennis (2013); Nguyen and Nguyen (2014) suggest and further confirm that financial markets are likely to be more correlated in period of burst than periods of booms. When dependence structure of financial markets (stock, bond, exchange rates and money markets) are closely related, they tend to be faced with a possibility of a market crash.

Until recently, extreme events (financial crisis) were regarded as outliers and often excluded from statistical analysis of financial market (Wu et al., 2012). The financial turmoil has highlighted the importance of analysing extreme events in investment decision, pricing of financial assets as well as risk management (Aloui et.al, 2011). Therefore, extreme events are likely to alter the dependence structure of financial markets. This could have implications for investment decisions and ability to estimate the risk of financial markets crash.

Market crashes are considered catastrophic events when the values of equity market suddenly decline, exchange rates depreciate rapidly and there is a credit default. These rare events could lead to instability of the financial system and exposure to systemic risk. In recent times, extreme events in the financial market are no longer considered as outlier with negligible probability.

Analysing extreme events with a normality assumption might be misleading (Chen et al., 2004). Restriction to elliptical distributions, implies dealing with measures which only captures dependence in the linear sense (Aloui et.al., 2011). Linear correlation models however, from empirical literature have been found not to be appropriate for measuring non normal distributions (Nelsen, 2006). Since financial market data exhibit heavy tails as a result, linear correlation models cannot capture the structure of dependence (Embrechts et al., 2002). Financial decision based on linear correlation models may be misleading as it is not robust in modelling of nonlinear dependence (Boyer et al., 1999).

Linear correlation models can lead to underestimation of the risks that could be associated with a financial market crash; thus it is pertinent to use models which capture financial market non-linearity. The aim of this paper is to estimate 
dependence structure and estimate the risk of market crash.

The remainder of the paper is organized as follows: section 2 gives the theoretical framework. Empirical results are analysed and discussed in Section 3. Conclusion and policy implications are provided in Section 4.

\section{Theoretical Framework}

Assume a portfolio of two financial market index. The initial value of the portfolio from Carmona (2004) is given as

$$
P_{0}=n_{1} A_{1}+n_{2} A_{2}
$$

where $n_{1}$ and $n_{2}$ are the number of units of the two financial markets index, which are valued at $A_{1}$ and $A_{2}$ at the beginning of a period. We denote $A_{1}^{\prime}$ and $A_{2}^{\prime}$ their values at the end of the period. The new value of this portfolio at the end of the new period is given by

$$
P_{1}=n_{1} A_{1}^{\prime}+n_{2} A_{2}^{\prime}
$$

to get log returns on the individual financial market index, we denote

$$
X=\log \left(\frac{A_{1}^{\prime}}{A_{1}}\right)
$$

and

$$
Y=\log \left(\frac{A_{2}^{\prime}}{A_{2}}\right)
$$

The $\log$ return of the portfolio is given from equation 2 is given by

$$
\begin{aligned}
=n_{1} A_{1} e^{x}+n_{2} A_{2} e^{y} & \\
R=\log \left(\frac{A}{A_{0}}\right) & =\log \left(\frac{n_{1} A_{1}}{n_{1} A_{1}+n_{2} A_{2}} e^{X}+\frac{n_{1} A_{1}}{n_{1} A_{1}+n_{2} A_{2}} e^{Y}\right) \\
& =\log \left(\lambda_{1} e^{X}+\lambda_{2} e^{Y}\right)
\end{aligned}
$$

where $\lambda_{1}$ and $\lambda_{2}$ are the individual market index.

We apply copula model to estimate the structure of dependence since linear correlation models are not capable to model dependence structure. In order to apply copula model to estimate the risk of financial market crash, we filter our log returns to obtain independent and identically distributed (i.i.d) data using the $\mathrm{GARCH}(1,1)$ model. The GARCH filtering provides us with a standardized residual of returns series which is use in estimating the marginal distribution. The standard GARCH $(\mathrm{p}, \mathrm{q})$ model by Bollerslev (1986) is given by

$$
\sigma_{t}^{2}=\omega+\sum_{i=1}^{q} \alpha_{i} \quad \varepsilon_{t-i}^{2} \sum_{j=1}^{p} \beta_{j} \sigma_{t-j}^{2}
$$

$\sigma_{t}^{2}$ is conditional variance and $\varepsilon_{t}$ is the innovation or residual returns defined as $\varepsilon_{t}=\sigma_{t} e_{t}, e_{t} \sim N(0,1)$ are standardized residual returns.

To measure dependence among the financial market returns, the filtered residuals are joined together applying copula function modelling. The joint distribution function of the random variable $X$ and $Y$ is given by

$$
F_{X Y}(x, y)=P_{r}(X \leq x, Y \leq y)
$$

Using the theorem of Sklar (1959), gives us a connection between marginal distribution and copulas to the joint distribution. In this case, let $F_{X Y}$ represent a bivariate cumulative distribution function with marginal distribution $F_{X}$ and $F_{Y}$, then there exist a two dimensional copula cumulative distribution function $C$ on $[0,1]^{2}$, such that for all $(x, y) \in \mathbb{R}^{2}$ $F_{X Y}(x, y)=C\left(F_{X}(x), F_{Y}(y)\right)$ holds. For continuous $F_{X}$ and $F_{Y}, C$ is uniquely determined by

$$
C(u, v)=F_{X Y}\left(F_{x}^{-1}(u), F_{Y}^{-1}(v)\right)
$$

the random variables $u=F_{X}(x)$ and $v=F_{Y}(y)$, are obtained by the probability integral transformation uniformly distributed on $[0,1]$, where $F_{Y}^{-1}(u)$ and $F_{X}^{-1}(v)$ are the generalised inverse distribution functions of marginal.

The Joint density function of (X Y) from Sklar (1959) is given by

$$
f(x, y)=C\left(F_{X}(x), F_{Y}(y)\right) f_{X}(x), f_{Y}(y)
$$

Literature offers several copulas such as Gaussian, Gumbel and Clayton which can be used in modelling dependence structure of a relationship described above (Kjersti, 2004). However, of interest to this study is the Clayton Copula. The Clayton copula is an asymmetric copula, exhibiting greater dependence in the negative tail than in the positive. Mathematically, the bivariate Clayton copula is expressed as 


$$
C_{\theta}(u, v)=\left(u^{-\theta}+v^{-\theta}-1\right)^{-\frac{1}{\theta}}
$$

its generator is

$$
\varphi_{\theta}(X)=\frac{1}{\theta}\left(x^{-\theta}-1\right)
$$

Where $u$ and $v$ are random variables, $0<\theta<\infty$ is a parameter controlling the dependence. Perfect dependence is obtained if $\theta \rightarrow \infty$, while $\theta \rightarrow 0$ implies independence. Therefore, markets crashing jointly can be modelled using the Clayton approach which is the main focus area of this research as it can tell about market risk in periods of extreme financial events.

\section{Estimating Copula Parameters}

We use the inference function for margins method (IFM) to fit copula and estimate the structure of dependence. The IFM is based on the pioneering work of (Joe and $\mathrm{Xu}, 1996)$. The estimation method of IFM is presented below:

Assume we observe $n$ independent observations $X_{t}=\left(x_{t 1}, x_{t 2}, \ldots, x_{t p}\right)$ from a multivariate distribution, which can be constructed with $p$ marginal distributions and a copula function $C\left(F_{1}(x), \ldots, F_{n}(x) ; \theta\right)$ with parameter $\theta$. The probability distribution function (PDF) of the marginal distributions is defined as $f_{i}\left(x ; \theta_{i}\right)$ with a cumulative density distribution (CDF) as $F_{i}\left(x ; \theta_{i}\right)$, where $\theta_{i}$ is the parameter of marginal distributions. The IFM method estimates the parameters of the marginal distribution in the first step.

The log-likelihood function of the first step can be written as

$$
\operatorname{Logl}(\theta)=\sum_{i=1}^{n} \quad \sum_{j=1}^{p} \log f_{i}\left(x_{i j} ; \theta_{i}\right)
$$

The estimation of the parameter $\theta=\left(\theta_{1}, \ldots, \theta_{n}\right)$ of marginal distribution can be made through maximizing the log-likelihood function (Joe and Xu, 1996).

$$
\hat{\theta}_{i}=\operatorname{argmax} \sum_{i=1}^{n} \quad \sum_{j=1}^{p} \log f_{i}\left(x_{i j} ; \theta_{i}\right)
$$

The parameter $\theta$ of the copula function is estimated in the second step of IFM, with the parameter $\hat{\theta}$ of the p marginal distributions.

$$
\hat{\theta}=\operatorname{argmax} \sum_{t=1}^{n} \log C\left(F_{1}\left(x_{i 1} ; \hat{\theta}_{i}\right), \ldots, F_{p}\left(x_{i p} ; \hat{\theta}_{p}\right) ; \theta\right)
$$

The IFM is given by a vector $\theta^{I F M}=\left(\hat{\theta}, \hat{\theta}_{I F M}\right)$

$$
\text { where } \hat{\theta}_{I F M}=\left(\hat{\theta}_{i}, \hat{\theta}_{p}, \theta\right)
$$

\section{Asymptotic Properties of Inference Function for Margin Estimator}

We use theorem 1 (Joe, 2005) to show consistency and asymptotic normality of the IFM estimator $\hat{\theta}_{I F M}$.

Theorem 1. Let $X_{1}, \ldots, X_{n}$ be independent and identically distributed random vectors with density $f_{\theta}$. Let $\theta \in \Theta$ and $x \in S:=\operatorname{supp}\left(f_{0}\right) \subseteq R^{2}$ where $\operatorname{supp}\left(f_{0}\right)$ is the support of $\left(f_{0}\right)$. Assuming the following conditions hold:

(a) The parameter space $\Theta \subseteq \mathrm{R}$ is an open interval

(b) The Support $S$ is independent of $\theta$

(c) $f(x ; \theta)$ is three times continuously differentiable with respect to $\theta$

(d) $E l_{\theta}(X: \theta)^{2}+E l_{\theta \theta}(X: \theta)=0$ and $\left.\int_{s} \frac{\partial}{\partial \theta} f(x: \theta) d x=\frac{\partial}{\partial \theta} \int_{s} f(x: \theta) d x=0\right)$

(e) The fisher Information $I(\theta)=E l_{\theta}(X: \theta)^{2}=-E l_{\theta \theta}(X: \theta)$ is positive and finite

(f) For all $\theta_{0} \in \Theta$ and $\theta \in \Theta$ and $\theta \in U_{\delta}\left(\theta_{0}\right)$ there exists a measurable function $M_{\theta_{0}}$ with $E_{\theta_{0}}\left(M\left(X: \theta_{0}\right)\right)<$ $\infty$ such that $\left|l_{\theta \theta \theta}(y: \theta)\right| \leq M\left(x: \theta_{0}\right)$ for all $x \in$.

Imposing the regularity conditions from (White 1994 and Patton 2006b) to the marginal likelihood in equation (12), and the copula likelihood function, equation (14), a joint normality condition holds such that as $n \rightarrow \infty$,

$$
\sqrt{n}\left(\left(\hat{\theta}_{I F M}\right)-\theta\right) \rightarrow N(0, \widehat{G})
$$

where $\widehat{G}$ is the estimator of the Godambe Information matrix (Joe, 1997).

$\mathrm{G}$ is defined as $G=\left(D_{g}{ }^{-1} M D_{g}{ }^{-1}\right)^{t}$, where $D_{g}=E\left(\frac{\partial g^{t}(X, \eta)}{\partial \eta}\right)$ and $M_{g}=E\left(g^{t}(X, \eta) g(X, \eta)\right)$.

\section{Estimating Market Risk}

In estimating financial market risk, the literature offers several risk measures. However, we reviewed Value at Risk (VaR). 
Value-at-Risk measures the minimum loss we would expect over a given time horizon. Carmona (2004) defined $V a R$ as the 100th percentile of the loss distribution given as:

$$
q=\mathbb{P}\{-R \geq r\}=\mathbb{P}\{R \leq-r\}=F_{R}(-r)
$$

where $q$ is the percentile, $\mathbb{P}$ is the probability $R$ is the random variable and $r$ is the losses.

From our earlier setting of a portfolio with two financial index and $\log$ returns denoted by $X$ and $Y$, we solve for $r$ in equation (15) to get our VaR with our copula parameter inputted in the VAR (Copula-VAR) by computing the CDF of the $\log$ return $R$. The latter can be expressed analytically as

$$
=\iint_{\left\{(x, y) ; \lambda_{1} e^{x}+\lambda_{2} e^{y}\right\} \leq e^{-r}} f(X, Y)^{(x, y) d x d y}
$$

where $\lambda_{1} e^{x}+\lambda_{2} e^{y}$ are log returns of the portfolio from equation (5) and $f(X, Y)$ is the CDF of the returns.

$$
\begin{aligned}
& =\int_{-\infty}^{-r-\log \lambda_{1}} d x \int_{-\infty}^{\log \left(e^{-r} / \lambda_{2}-\lambda_{1} / \lambda_{2} e^{x}\right)} c\left(F_{X}(x), F_{Y}(y)\right) f_{X}(x) f_{Y}(y) d y \\
& =\int_{0}^{F_{X}\left(-r-\log \lambda_{1}\right)} d u \int_{0}^{F_{Y}\left(\log \left(e^{-r} / \lambda_{2}-\lambda_{1} / \lambda_{2} e_{X}^{F^{-1}(u)}\right)\right)} d v c(u, v) \\
& \left.\left.=\left.\int_{0}^{F_{X}\left(-r-\log \lambda_{1}\right)} d u \frac{\partial}{\partial u} C(u, v)\right|_{v=F_{Y}\left(\operatorname { l o g } \left(e^{-r} / \lambda_{2}-\lambda_{1} / \lambda_{2} e_{X}^{F^{-1}}(u)\right.\right.}\right)\right)
\end{aligned}
$$

The steps for inputting copula parameter into VaR is given in Appendix I.

In order to estimate the VaR in the given framework, we estimate the copula dependence parameter from a sample of pairs of log returns. The estimated parameter is then used to compute the VaR quantiles at different confidence levels which gives estimates for VaR.

\section{Empirical Results}

In the analysis of financial market dependence, monthly data were collected from January, 2000 to March, 2016 from stock exchange websites of countries namely; Nigeria, Kenya and South Africa. Consideration for proxy is All Share Index. Data was divided into three periods; Pre-crisis, crisis and Post crisis periods which captured financial market extreme events (2007-2009). We used negative log returns obtained as:

$$
r_{t}=-\operatorname{In}\left(\frac{A_{t}}{A_{t-1}}\right)
$$

where $A_{t}$ is today's index and $A_{t-1}$ is the previous day's index.

The summary statistics for each log return index are reported in Table 1. A glance at the results from the Jarque-Bera test, reveals none of series returns are normally distributed. Figure 1 gives the trend and log returns of each index.

Table 1. Monthly Summary Statistics for Each Index

\begin{tabular}{cccccc}
\hline Index & Mean & Standard Deviation & Skewness & Kurtosis & Jarque-Bera \\
\hline NSE20 & 0.0026 & 0.0404 & -0.474 & 2.502 & $p<0.00005$ \\
NASI & 0.0076 & 0.0719 & -0.509 & 5.360 & $p<0.00005$ \\
JSE & 0.0097 & 0.0490 & -0.3149 & 0.563 & $p<0.00005$ \\
\hline
\end{tabular}

Table 1 gives a summary statistic for the negative log returns of Kenya, Nigeria and South Africa stock markets. The p-values of Jarque-Bera normality test are shown in the last column. The sample period on a monthly basis covers from January 2000 to March 2016. Observations are 195, collected from various stock exchange websites. NSE20-Kenya Stock Market Index, NASI; Nigerian Stock Market Index and JSE: South African Stock Index. 

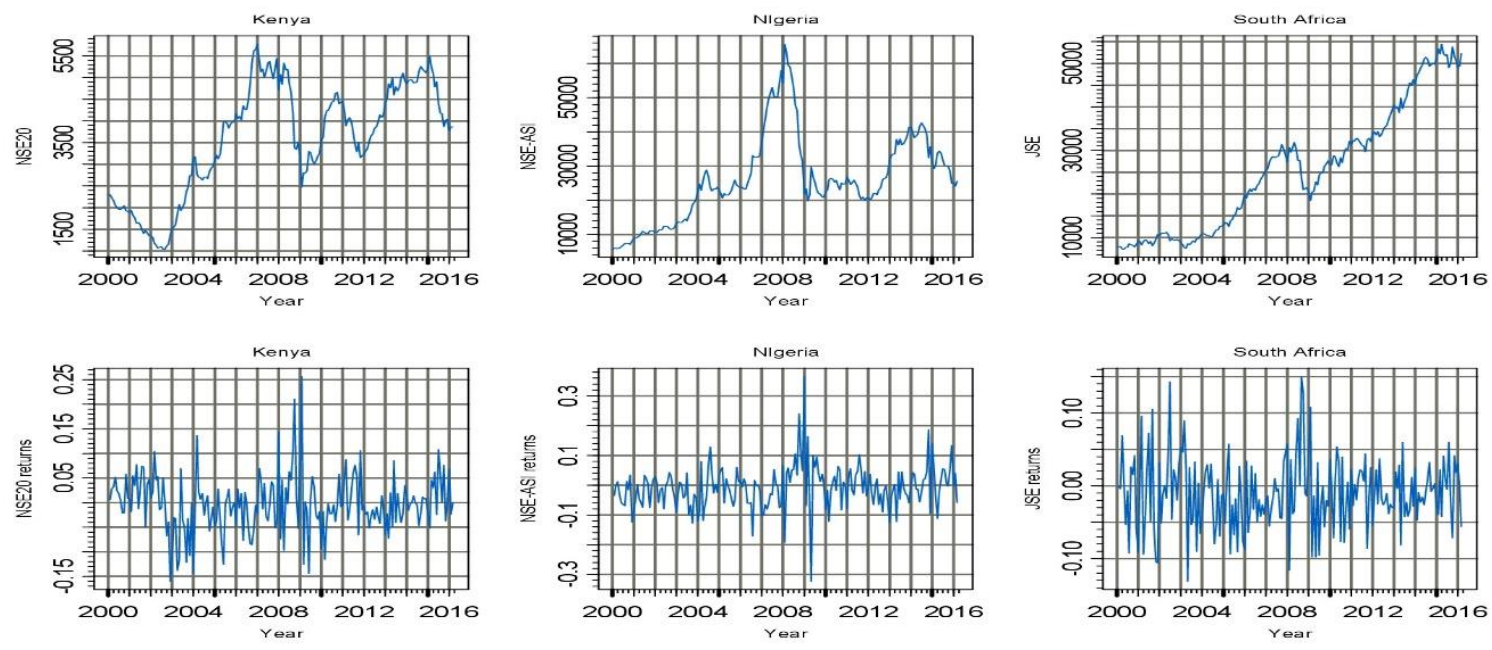

Figure 1. Monthly Stock Market Evolution and Log Returns

Figure 1 reveals that stock market index for Kenya, Nigeria and South Africa was trending upwards which can be described as a pre-crisis period from around 2003 to 2006 for Kenya, 2000 -2006 for Nigeria and 2000-2006 for South Africa. Between 2007-2009 a downward trend is seen for all index (crisis) and from 2010-2016 a post crisis period.

\section{Copula Fitting}

We report results from fitting Clayton copula. Figure 2 gives a visual display of the dependence between market pairs.
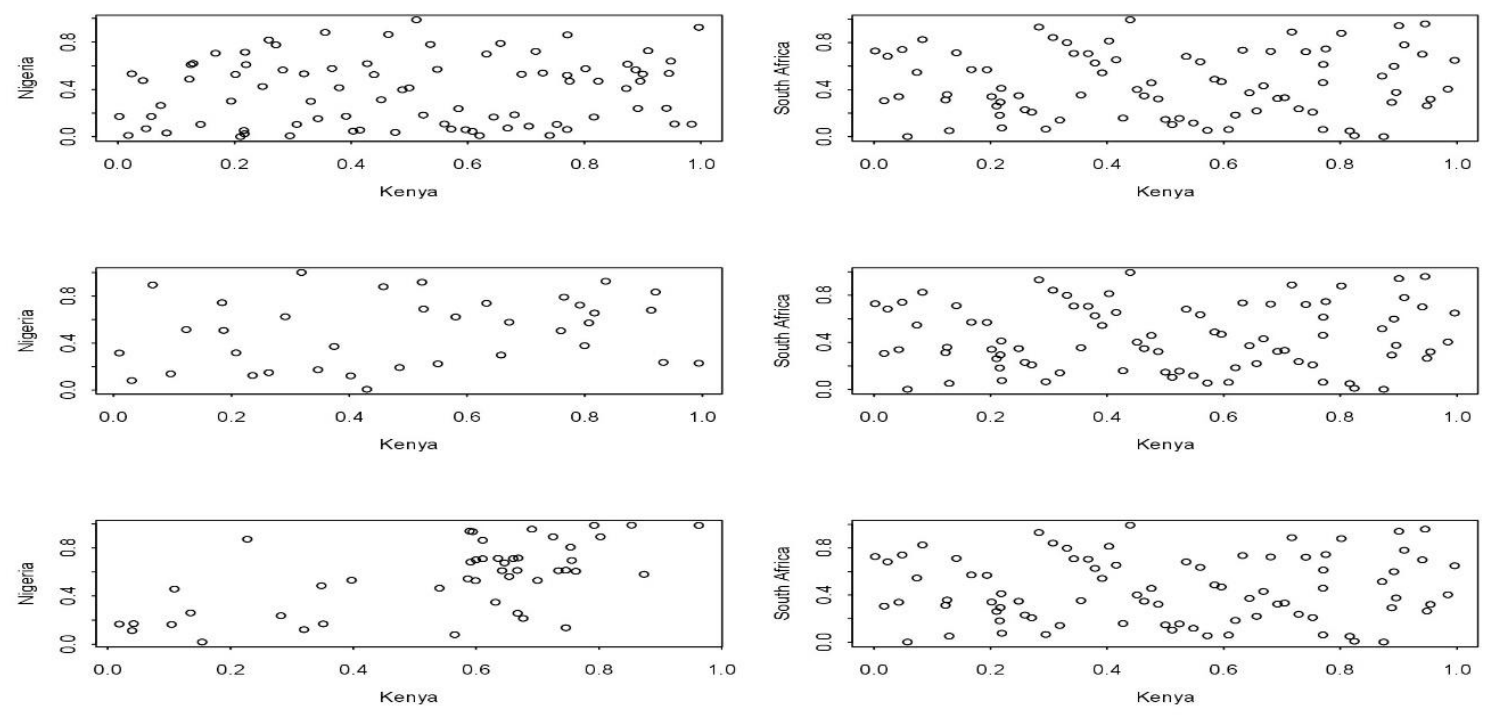

Figure 2. Scatter plots of Stock Market Pairs

The first row gives a scattered plot for the fitted copula, showing patterns of dependence between Kenya and Nigeria stock market between 2000-2006 and Kenya and South Africa in the same period. The second row in a similar manner shows the scattered plot for Kenya and Nigeria between 2007-2009 and Kenya South Africa Between 2007-2009. The observation shows a dependence originating from the low side of the plot. Third row gives the scatter plot for Kenya-Nigeria and Kenya-South Africa for the period 2010-2016. 

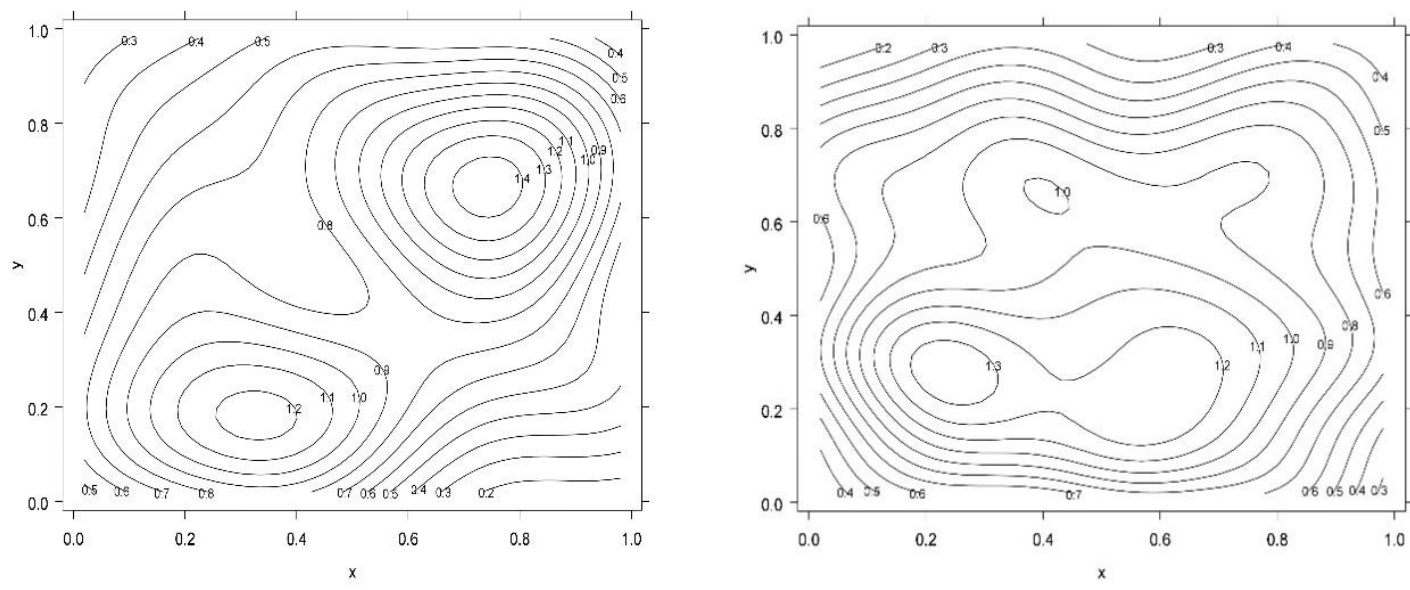

Figure 3. Contour Plot of Clayton Copula (Crisis Period)

Figure 3 gives us a contour plot for Kenya-Nigeria pair on the left and for Kenya-South African pair on the right.

Table 2 gives estimates from fitting clayton copulas to stock market pairs for a period considered as pre crisis between 2000-2006. The table reports the parameter of the copula and also the linear parameters estimates of the relationship between these pairs.

Table 2. Empirical Fitting of Copula 2000-2006 (Pre Crisis Period)

\begin{tabular}{cccccc}
\hline \multirow{5}{*}{ Kenya -Nigeria (Market Pair) } \\
Copula & $\theta$ & LogLike & AIC & Kendall's & Spearman \\
\cline { 2 - 6 } Clayton & 0.1574 & 0.8471 & 0.3048 & 0.0729 & 0.1670 \\
& $(0.1374)$ & & & \\
\hline \multirow{7}{*}{ Kenya - South Africa (Market Pair) } \\
Copula & $\theta$ & LogLike & AIC & Kendall's & Spearman \\
\cline { 2 - 6 } Clayton & 0.09943 & 0.36285 & 0.1673 & 0.0473 & 0.0709 \\
& $(0.1259)$ & & & \\
\hline
\end{tabular}

Table 2 gives a summary of copula fit for Kenya-Nigeria stock market index pair and Kenya-South Africa stock market index pair. $\theta$ is the dependence parameter for clayton copula during the pre-crisis period. The Kendall's tau reports a dependence of 0.072 and Spearman rho of 0.167 for Kenya-Nigeria stock market pair, while a dependence of 0.0473 and 0.070 for Kendall's tau and Spearman are reported for Kenya-South Africa market pair.

Table 3. Copula Fitting for 2007-2009 (Crisis Period)

\begin{tabular}{|c|c|c|c|c|c|}
\hline \multirow{3}{*}{ Copula } & \multicolumn{5}{|c|}{ Kenya -Nigeria (Market Pair) } \\
\hline & \multicolumn{5}{|c|}{ Parameter } \\
\hline & $\theta$ & LogLike & AIC & Kendall's & Spearman \\
\hline \multirow[t]{2}{*}{ Clayton } & $0.2863(0.2486)$ & 0.9114 & 0.1771 & 0.1252 & 0.1865 \\
\hline & \multicolumn{5}{|c|}{ Kenya - South Africa (Market Pair) } \\
\hline \multirow{2}{*}{ Copula } & \multicolumn{5}{|c|}{ Parameter } \\
\hline & $\theta$ & LogLike & AIC & Kendall's & Spearman \\
\hline Clayton & $0.9201(0.3189)$ & 6.6607 & -11.3215 & 0.3151 & 0.4542 \\
\hline
\end{tabular}


Table 3 gives an overview of fitting clayton copula to stock market pairs in an extreme period considering the 2007-2009 period. In this period, the dependence parameter $\theta$ between Kenya -Nigeria stock market index is 0.286 and for Kenya-South Africa market index is 0.9201 . The Kendall's tau reports a dependence of 0.125 and Spearman rho of 0.186 for Kenya-Nigeria stock market pair, while a dependence of 0.315 and 0.452 for Kendall's tau and Spearman are reported for Kenya-South Africa market pair.

Table 4. Copula Fitting for 2010-2016 (Post Crisis Period)

\begin{tabular}{|c|c|c|c|c|c|}
\hline \multirow{3}{*}{ Copula } & \multicolumn{4}{|c|}{ Kenya -Nigeria (Market Index Pair) } & \\
\hline & \multicolumn{5}{|c|}{ Parameter } \\
\hline & $\theta$ & LogLike & AIC & Kendall's & Spearman \\
\hline \multirow[t]{2}{*}{ Clayton } & $0.4307(0.1847)$ & 3.328 & -4.657 & 0.2536 & 0.3727 \\
\hline & \multicolumn{4}{|c|}{ Kenya - South Africa (Market Index Pair) } & \\
\hline \multirow{2}{*}{ Copula } & \multicolumn{5}{|c|}{ Parameter } \\
\hline & $\theta$ & LogLike & AIC & Kendall's & Spearman \\
\hline Clayton & $0.3469(0.159)$ & 3.1707 & -4.3414 & 0.1478 & 0.2196 \\
\hline
\end{tabular}

In table 4, we report the fitting of clayton copula to stock market pairs in the post crisis period. In this period, the dependence between Kenya-Nigeria stock market pair is 0.430 and for Kenya -South Africa is 0.346 . The Kendall's tau reports a dependence of 0.253 and Spearman rho of 0.37 for Kenya-Nigeria stock market pairs, while a dependence of 0.147 and 0.219 for Kendall's tau and Spearman are reported for Kenya-South Africa.

\section{Estimating Market Risk}

After estimation of copula parameter $\theta$, we substituted the dependence parameter into the VaR functions to estimate risk measures at $90 \%, 95 \%$ and $99 \%$ using the corresponding quantiles 0.10 .0 .05 and 0.01 respectively. The VaR estimates are used in measuring the maximum possible loss of market value over a holding period. The VaR is computed as 0.759 at $90 \%, 0.784$ at $95 \%$ and 0.837 at $99 \%$ confidence interval which implies the losses from holding the Kenya-Nigeria portfolio is $75.9 \%, 78.4 \%$ and $83.7 \%$ at the respective confidence interval as shown in table 5. Simultaneously, VaR estimates computed for Kenya - South Africa portfolio reveal a 0.776 at $90 \%, 0.795$ at $95 \%$ and 0.855 at $99 \%$ confident interval which implies the losses from holding the Kenya-South Africa portfolio is $77.6 \%, 79.5 \%$ and $85.5 \%$ at the respective confidence interval. Since a higher VaR value implies a higher risk, this indicates that holding a Kenya-South Africa portfolio is riskier than holding a Kenya-Nigeria portfolio.

Table 5. Value at Risk Estimates Using Clayton Copula

\begin{tabular}{cccc}
\hline & \multicolumn{2}{c}{ Crisis Period: Clayton Copula } \\
& $90 \%$ VaR & VaR 95\% & VaR99\% \\
NSE20-NASI & 0.759 & 0.784 & 0.837 \\
\hline & $90 \%$ VaR & VaR 95\% & VaR 99\% \\
NSE20-JSE & 0.776 & 0.795 & 0.855 \\
\hline
\end{tabular}

\section{Discussion and Conclusion}

The study focused on stock markets (Kenya, Nigeria and South Africa) using copula technique which estimates dependence structure of stock markets during pre-crisis, crisis and post-crisis periods. Clayton copula dependence parameters have been estimated using the Inference function for margins method into the VaR framework. The results revealed that during the crisis period, the maximum possible loss of market value is $75.9 \%$ and $77.6 \%$ with a confident interval of $90 \%$ for the Kenya-Nigeria and Kenya-South Africa portfolios respectively. This implies that the Kenya-South Africa portfolio has the highest risk. A further implication is that dependence during crisis period imply that opportunities for portfolio diversification are reduced than at periods of booms.

\section{References}

Aloui, R., Aïssa, M. S. B., \& Nguyen, D. K. (2011). Global financial crisis, extreme interdependences, and contagion effects: The role of economic structure?. Journal of Banking \& Finance, 35(1), 130-141. http://dx.doi.org/10.1016/j.jbankfin.2010.07.021

Boyer, B. H., Gibson, M. S., \& Loretan, M. (1997). Pitfalls in tests for changes in correlations (Vol. 597). Board of 
Governors of the Federal Reserve System.

Carmona. (2004). Statistical Analysis of Financial Data in S-Plus. Springer Verlag. http://www.princeton.edu/rcaroma/safd

Chen, Fan, Y., \& Patton, A. (2004). Simple Test for Models of Dependence between Multiple Financial Time Series, with Application to U.S. Equity Returns and Exchange Rates). London Economics Financial market group, 43(483).

Dennis, E. (2013). Developing country vulnerability in light of the global financial crisis: Shock therapy. Review of Development Finance, 3, 61-83. http://dx.doi.org/10.1016/j.rdf.2013.02.001

Embrechts, P., McNeil, A., \& Straumann, D. (2002). Correlation and dependence in risk management: Properties and pitfalls. Cambridge University Press, 176-223. http://dx.doi.org/10.1017/cbo9780511615337.008

Joe, H., \& Xu, J. (1996). The Estimation Method of Inference Functions for Margins for Multivariate Models. Department of Statistics, University of British Columbia, (166).

Joe, H., (1997). Multivariate Models and Dependence Concepts. Chapman \& Hall, London. http://dx.doi.org/10.1201/b13150

Joe, H., (2005). Asympotic Efficiency of the Two Stage estimation method for Copula based models. Journal of multivariate Analysis 94 (2005), 401-419. http://dx.doi.org/10.1016/j.jmva.2004.06.003

Kjersti, A. (2004). Modelling the dependence structure of financial assets: a survey of four copulas. Applied research and development.

Ling, H. (2006). Dependence patterns across financial markets: A mixed copula approach. Applied Financial Economics, 717-729.

Nelsen, R. (2006). An introduction to copulas. Computational Statistics and Data Analysis, 6, 272.

Nguyen, C., \& Nguyen, T. (2014). Analysing Dependence Structure of Equity, Bond and money Market by using time varying Copula. International Journal of Economics and Finance, 6(3), 787-815. http://dx.doi.org/10.5539/ijef.v6n3p37

Patton, A. (2006b). Modelling asymmetric exchange rate dependence. International Economic Review, 47(2), 527-556. http://dx.doi.org/10.1111/j.1468-2354.2006.00387.x

Poon, S., M, M. R., \& Tawn, J. (2004). Extreme Value Dependence in Financial Markets: Diagnostics, Models, and Financial Implications. Review of Financial Studies, 17, 581-610. http://dx.doi.org/10.1093/rfs/hhg058

Sklar, A. (1959). Fonctions de repartitiona $n$ dimensions et leurs marges. Publication de l'Institute de Statistique de l'Universite de Paris, 8.

Vaart A. W., (2000). Asymptotic Statistics. Cambridge University Press.

White H. (1994). Estimation, Inference and Specification Analysis. Econometric Society Monographs No. 22. Cambridge University Press: Cambridge. http://dx.doi.org/10.1017/CCOL0521252806

Wu, Zhang, Z., \& Zhao, Y. (2012). Study of the Tail Dependence Structure in Global Financial Markets Using Extreme Value Theory. Journal of Reviews on Global Economics, 1, 62-81.

\section{Appendix A}

\section{Copula VaR}

We show how we input $\theta$, the copula parameter into VaR. From the definition of VaR. Carmona (2004) defined $V a R_{q}$ as the $100_{q}$-th percentile of the loss distribution given as:

$$
q=\mathbb{P}\{-R \geq r\}=\mathbb{P}\{R \leq-r\}=F_{R}(-r)
$$

We solve for $r$ in equation (15) to get our VaR, we computing the CDF of the log return $R$. The latter can be expressed analytically as

$$
=\iint_{\left\{(x, y) ; \lambda_{1} e^{x}+\lambda_{2} e^{y}\right\} \leq e^{-r}} f(X, Y)^{(x, y) d x d y}
$$

Now for a continuous case, the continuous distribution function (CDF) is given by 


$$
F(X, Y)=\int_{-\infty}^{x} \int_{-\infty}^{y} f(a, b) d a d b
$$

Note: For double integral, we assume that one variable is constant and the other is varying. So here we assume $X$ to be the only variable and $Y$ as a constant. The above generates the upper limit of $X$ as follows.

$$
\log \left(\lambda e^{x}+\lambda e^{Y}\right) \leq-r \Rightarrow \lambda e^{x}+\lambda e^{Y} \leq e^{-r}
$$

When $Y$ is considered a constant, we then have

$$
\lambda_{1} e^{X} \leq \lambda_{1} \leq \frac{e^{-r}}{e^{x}} \Rightarrow \lambda_{1} \leq e^{-r-X} \Rightarrow \log \left(\lambda_{1}\right) \leq-r-X \leq-r-\log \lambda_{1}
$$

Hence, the upper limit for $X$ is $-r-\log \lambda_{1}$

Then for $Y$, we vary both $x$ and $Y$. This is because we cannot assume $X$ to be constant as we have already assumed $Y$ to be one but now we integrate with respect to $X$.

So we have,

$$
\lambda_{1} e^{X}+\lambda e^{Y} \leq e^{-r} \Rightarrow \frac{\lambda_{1} e^{X}}{\lambda_{2}}+\frac{\lambda_{2} e^{X}}{\lambda_{2}} \leq \frac{e^{-r}}{\lambda_{2}}-\frac{\lambda_{1} e^{X}}{\lambda_{2}} \Rightarrow Y \leq \log \left(\frac{e^{-r}}{\lambda_{2}}-\frac{\lambda_{1}}{\lambda_{2}}\right)
$$

As the upper limit for $Y$. Which gives of the equation (17) below

$$
=\int_{-\infty}^{-r-\log \lambda_{1}} d x \int_{-\infty}^{\log \left(e^{-r} / \lambda_{2}-\lambda_{1} / \lambda_{2} e^{x}\right)} c\left(F_{X}(x), F_{Y}(y)\right) f_{X}(x) f_{Y}(y) d y
$$

\section{Change of variables and integration}

Now, we do a change of variables so as to integrate in a mathematically correct way. So recall that for copulas, $F\left(x_{1}, x_{2}, \ldots, x_{n}\right)=C\left(F_{1}\left(x_{1}\right), F_{2}\left(x_{2}\right), \ldots, F_{n}\left(x_{n}\right)\right)$ from Sklar Theorem (1959),

Recall also that

To bring in $u$, we do a transformation,

$$
u=f(X)
$$

Such that when $X=-\infty, u=0$ and when $X=-r-\log \lambda_{1}, u=F_{X}\left(-r-\log \lambda_{1}\right)$

For $v, v=f(Y)=F_{Y}(y)$ such that when $Y=-\infty, v=0$ and when

$$
\begin{gathered}
Y=\frac{e^{-r}}{\lambda_{2}}-\frac{\lambda_{1} e^{X}}{\lambda_{2}} \\
v=F_{Y}\left(\frac{e^{-r}}{\lambda_{2}}-\frac{\lambda_{1} e^{F_{X}^{-1}(u)}}{\lambda_{2}}\right) \\
=\int_{0}^{F_{X}\left(-r-\log \lambda_{1}\right)} d u \int_{0}^{F_{Y}\left(\log \left(e^{-r} / \lambda_{2}-\lambda_{1} / \lambda_{2} e_{X}^{F^{-1}(u)}\right)\right)} d v c(u, v)
\end{gathered}
$$

By integrating equation (18) we

$$
=\left.\int_{0}^{F_{X}\left(-r-\log \lambda_{1}\right)} d u \frac{\partial}{\partial u} C(u, v)\right|_{v=F_{Y}\left(\log \left(e^{-r} / \lambda_{2}-\lambda_{1} / \lambda_{2} e_{X}^{F^{-1}}(u)\right)\right.}
$$

\section{Copyrights}

Copyright for this article is retained by the author(s), with first publication rights granted to the journal.

This is an open-access article distributed under the terms and conditions of the Creative Commons Attribution license (http://creativecommons.org/licenses/by/4.0/). 